Article

\title{
Sensitivity Enhanced Photoacoustic Imaging Using a High-Frequency PZT Transducer with an Integrated Front-End Amplifier
}

\author{
Chen Yang ${ }^{1,2} \oplus$, Xiaohua Jian ${ }^{2}$, Xinle Zhu ${ }^{2}$, Jiabing Lv ${ }^{2}$, Yang Jiao ${ }^{2}$, Zhile Han ${ }^{2}$, \\ Antonios Stylogiannis ${ }^{3,4}$, Vasilis Ntziachristos ${ }^{3,4}$, George Sergiadis ${ }^{2,3,5}$ and Yaoyao Cui ${ }^{2, *}$ \\ 1 University of Science and Technology of China, Hefei 230026, China; yangc@sibet.ac.cn \\ 2 Suzhou Institute of Biomedical Engineering and Technology, Chinese Academy of Sciences, Suzhou 215163, \\ China; jianxh@sibet.ac.cn (X.J.); zhuxl@sibet.ac.cn (X.Z.); lvjb@sibet.ac.cn (J.L.); jiaoy@sibet.ac.cn (Y.J.); \\ hanzl@sibet.ac.cn (Z.H.); sergiadi@sibet.ac.cn (G.S.) \\ 3 Institute of Biological and Medical Imaging, Helmholtz Zentrum München, 85764 Neuherberg, Germany; \\ stylogiannis@helmholtz-muenchen.de (A.S.); v.ntziachristos@helmholtz-muenchen.de (V.N.) \\ 4 Chair for Biological Imaging, Technische Universität München, 81675 Munich, Germany \\ 5 School of Electrical and Computer Engineering, Aristotle University, 54124 Thessaloniki, Greece \\ * Correspondence: cuiyy@sibet.ac.cn
}

Received: 23 December 2019; Accepted: 26 January 2020; Published: 30 January 2020

\begin{abstract}
Photoacoustic (PA) imaging is a hybrid imaging technique that can provide both structural and functional information of biological tissues. Due to limited permissible laser energy deposited on tissues, highly sensitive PA imaging is required. Here, we developed a $20 \mathrm{MHz}$ lead zirconium titanate (PZT) transducer $(1.5 \mathrm{~mm} \times 3 \mathrm{~mm})$ with front-end amplifier circuits for local signal processing to achieve sensitivity enhanced PA imaging. The electrical and acoustic performance was characterized. Experiments on phantoms and chicken breast tissue were conducted to validate the imaging performance. The fabricated prototype shows a bandwidth of $63 \%$ and achieves a noise equivalent pressure (NEP) of $0.24 \mathrm{mPa} / \sqrt{\mathrm{Hz}}$ and a receiving sensitivity of $62.1 \mu \mathrm{V} / \mathrm{Pa}$ at $20 \mathrm{MHz}$ without degradation of the bandwidth. PA imaging of wire phantoms demonstrates that the prototype is capable of improving the detection sensitivity by $10 \mathrm{~dB}$ compared with the traditional transducer without integrated amplifier. In addition, in vitro experiments on chicken breast tissue show that structures could be imaged with enhanced contrast using the prototype and the imaging depth range was improved by $1 \mathrm{~mm}$. These results demonstrate that the transducer with an integrated front-end amplifier enables highly sensitive PA imaging with improved penetration depth. The proposed method holds the potential for visualization of deep tissue structures and enhanced detection of weak physiological changes.
\end{abstract}

Keywords: photoacoustic imaging; detection sensitivity; PZT transducer; front-end amplifier

\section{Introduction}

Photoacoustic (PA) imaging has drawn much attention worldwide during the last two decades with its compelling advantage of combining the high-contrast of pure optical imaging with the deep penetration depth of ultrasound (US) imaging [1-5]. Different from the conventional pulse-echo based US imaging, PA imaging is typically performed by illuminating the target with a nanosecond laser pulse and then detecting the ultrasonic signals using a mechanically scanned single-element ultrasonic transducer or an array probe [6-8]. PA imaging has been demonstrated to be capable of high resolution structural imaging such as mapping of subcutaneous micro-vessels [9-13], anatomic features discrimination [14-16], and detection of atherosclerotic plaques based on the 
lipid-specific contrast [17-19]. Meanwhile, PA imaging also shows the potential for physiological information measurements such as hemoglobin oxygen saturation (sO2) [20-22], metabolic oxygen rate [23], and tissue temperature distribution [24-26]. With the help of exogenous contrasts, PA molecular imaging and gene imaging has been achieved [27-31].

While PA imaging has been explored in a variety of biomedical applications, there is an extensive demand to improve the detection sensitivity [32-34]. Since the signal attenuation caused by the scattering of light and the attenuation of the acoustic waves is more than one order of magnitude per centimeter at $10 \mathrm{MHz}$ [35], the acoustic pressure of the PA signals arriving at the transducer is in the Pa or sub-Pa range depending on the depth [36]. When high frequency transducers ( $\geq 20 \mathrm{MHz}$ ) are used to achieve high spatial resolutions, signals become extremely weak considering the frequency-dependent attenuation on the order of $0.5 \mathrm{~dB} \cdot \mathrm{cm}^{-1} \cdot \mathrm{MHz}^{-1}$ in biological tissues [37]. Thus, high detection sensitivity is required in PA imaging. For a given absorption contrast, the detection sensitivity of PA imaging mainly depends on the incident light exposure and the efficiency of the detector [38]. As the laser energy increases, the enhancement of PA signal is eventually limited by the optical absorption saturation and the maximum permissible exposure specified by American National Standards Institute (ANSI) [39]. Therefore, highly sensitive acoustic detectors are of particular interest.

Optical and interferometric detectors like Fabry-Perot interferometers, are frequently utilized in PA imaging for all-optical light delivery and high sensitivity detection. However, they are difficult to form an array for fast imaging. Capacitive micromachined ultrasonic transducers (CMUTs) are also used for PA imaging because of their attractive large bandwidth and ease of miniaturization, but they are not readily available and a large DC bias is required to achieve high performance [40]. The most widely used detectors in PA imaging are the lead zirconium titanate (PZT) transducers due to their well-established technology, low cost of implementation, and high sensitivity [41]. However, there is a trade-off between the size and the sensitivity of the PZT transducer. The achieved sensitivity usually drops with reduced aperture and gets further degraded by the connecting cable, especially for high frequency transducers [42].

Integrating ultrasound transducer with front-end circuits is an efficient way to process signals locally and maintain the signal integrity. Transducer arrays with custom application-specified integrated circuits (ASIC) have been previously demonstrated in 3D ultrasound imaging [42-44]. However, these devices overwhelmingly operate with frequencies lower than $10 \mathrm{MHz}$, which are not enough for detecting the high frequency components of the PA signals. Their detection sensitivity also still requires further optimization for PA imaging. In addition, PA microscopic applications generally demand single-element high-frequency transducers with high sensitivity to detect signals generated by tiny biological structures [45].

In this work, we proposed a $20 \mathrm{MHz}$ PZT transducer with an integrated front-end amplifier for PA imaging with high sensitivity. The amplifier has an ultra-wide bandwidth beyond $200 \mathrm{MHz}$ and is capable of offering front-end signal amplification with a low noise figure while keeping the transducer sufficiently compact. The design, fabrication, and characterization of a prototype are presented in this work. Using the transducer with an integrated front-end amplifier, PA imaging experiments were performed on phantoms and chicken breast tissue in vitro to demonstrate the improvement of the signal-to-noise ratio (SNR) and the imaging depth range. High detection sensitivity achieved by the integrated transducer may enable a variety of pre-clinical and clinical applications that requires further in vivo evaluation. A preliminary version of this work has been reported [46].

\section{Materials and Methods}

\subsection{PZT Transducer with an Integrated Front-End Amplifier}

The front-end amplifier used is designed as a rectangular die with a highly compact structure such that simple auxiliary circuits are required for operation, as shown in Figure 1. Similar to typical 
implementation [47], only a bias tee composed of an inductor L and a capacitor C is necessary for DC bias of the amplifier and AC coupling to the coaxial cable.

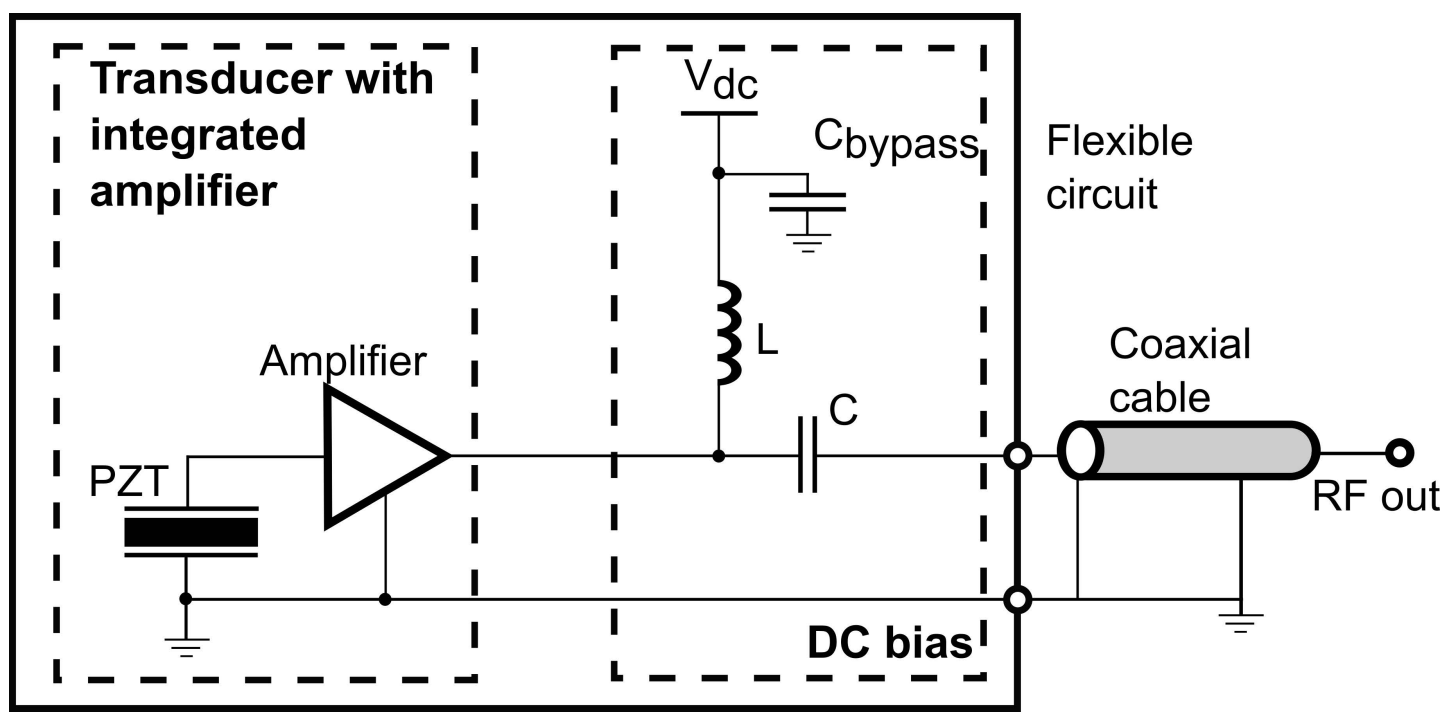

Figure 1. Circuit diagram of the integrated front-end amplifier circuits.

A front-end amplifier closely coupled to the PZT element firstly reduces the additional noise at the input of the amplifier by minimizing the electrical path between the transducer and the amplifier. Since the transmission line will introduce noise and interference to the signal, it is always crucial to place the amplifier to the transducer as close as possible to obtain a clean signal. Secondly, the front-end amplifier offers a high gain with a low noise figure to generate a strong enough signal to be noise-tolerant, such that the noise added by the following transmission lines will not significantly degrade the SNR.

The assembly of the proposed PZT transducer with an integrated amplifier die is illustrated in Figure 2a. A 0.3-mm thick flexible circuit provides the electrical connections for biasing and signal handling and serves as an intermediate connector between the transducer and the die. For proper grounding, the die was attached on a flat gold-coated plate on the rear side of the flexible circuit. The bond pads on the die were wire-bonded to the corresponding pads on the flexible circuit with 1-mil diameter gold wires. The miniature feature of the die $(820 \mu \mathrm{m} \times 760 \mu \mathrm{m})$ enables tight integration with transducers of various sizes. An unfocused PZT transducer with an active area of $1.5 \mathrm{~mm} \times 3 \mathrm{~mm}$ was built in house. The size of the transducer was determined to achieve optimal sensitivity and ease of fabrication. The fabricated PZT transducer was attached on the front side of the flexible circuit using conductive silver paste (H20E, EpoTek, Billerica, MA, USA). The cross section of the transducer with an integrated amplifier die is shown in Figure $2 b$.

A cooper plated via was drilled through the flexible circuit to connect the ground of the transducer and the die mounting plate. The top electrode of the transducer was interfaced to the input pad of the die by wire bonding. All bonding wires were kept as short as possible to reduce performance degradation caused by series inductance. Two 1-m long coaxial cables for signal output and DC power supply respectively, were soldered to the corresponding pads on the flexible circuit. Then the flexible circuit was inserted into a customized housing and fixed with an epoxy to reduce the interference from outside. After successful assembly of the transducer, a 14- $\mu \mathrm{m}$ parylene film (Parylene C, Special Coating Systems, Indianapolis, IN, USA) was coated on the transducer for acoustic impedance matching and electrical isolation. The fabricated prototype is shown in Figure 2c,d. For comparison, another PZT transducer of the same aperture and assembly but without the integrated amplifier was fabricated. In the rest of the paper, we referred to the transducer with and without an integrated amplifier as aUST and UST, respectively. 
(a)

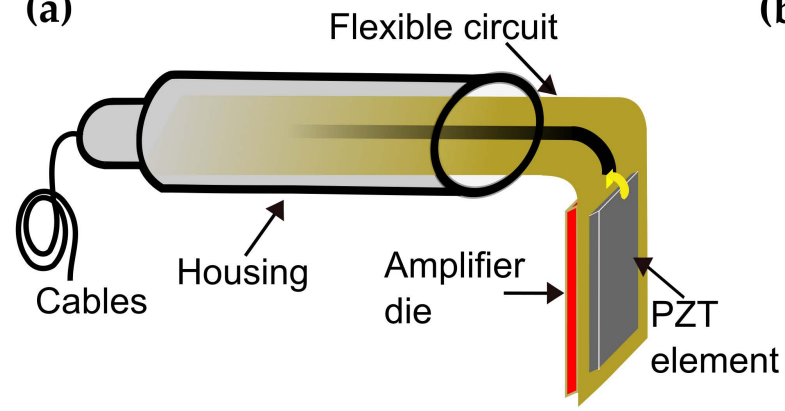

(b)

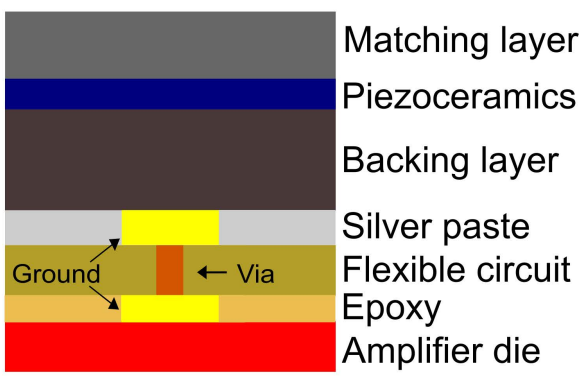

(c)

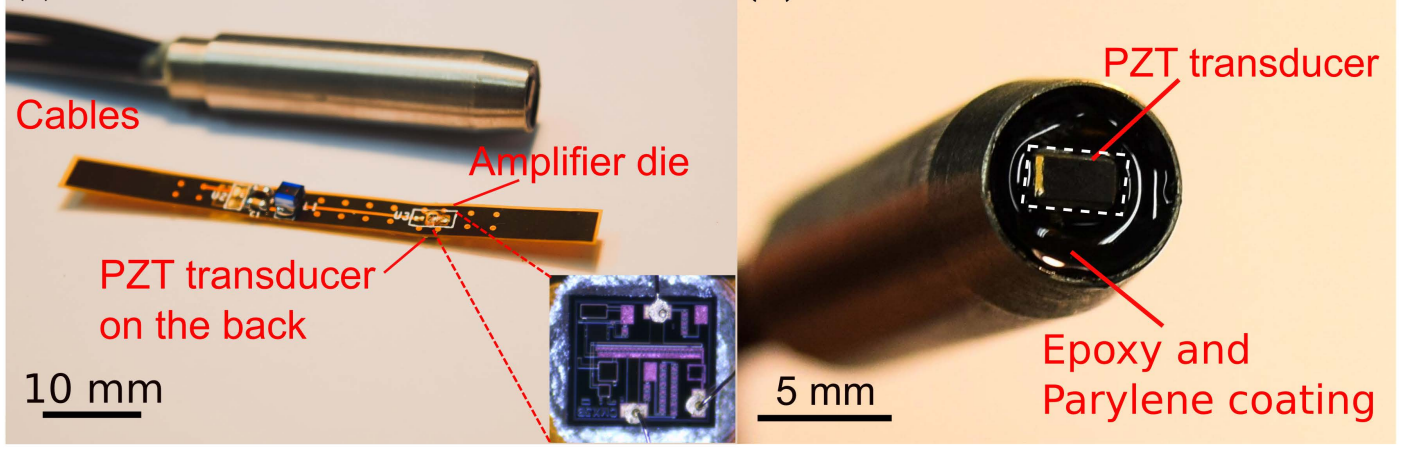

Figure 2. Schematic of the assembly of the transducer (a) and the cross section of the PZT transducer with an integrated front-end amplifier (b). (c) Photo of the fabricated prototype and the flexible circuit. Inset: photo of the amplifier die and bonding wires (d) PZT transducer on the tip.

\subsection{Transducer Characterization}

Before being attached on the flexible circuit, the electrical impedance of the fabricated PZT element was measured using an impedance analyzer (E991A, Keysight Technologies, Santa Rosa, CA, USA). The output frequency response of the proposed circuit shown in Figure 1 was measured using a network analyzer (E5061B, Keysight Technologies, Santa Rosa, CA, USA). The signal source was applied to the input of the amplifier via a capacitor with an impedance comparable to that of the fabricated PZT element. The output noise spectrum of the transducer with an integrated amplifier was achieved by computing the power spectral density of the measured noise.

To characterize the receiving sensitivity of the fabricated transducer, a $20 \mathrm{MHz}$ unfocused transducer was used as a transmitter and was driven by a function generator (33250A, Keysight Technologies, Santa Rosa, CA, USA) to transmit acoustic waves, as shown in Figure 3. The UST and the aUST were mounted on a 3D stage and positioned at $\sim 3 \mathrm{~mm}$ away from the transmitter to receive the acoustic signals, respectively. The output signals were averaged 16 times and recorded by an oscilloscope (DPO5034, Tektronix, Beaverton, OR, USA) for offline analysis. To calibrate the acoustic pressure of the transmitted waves, a PVDF needle hydrophone with a diameter of $0.2 \mathrm{~mm}$ (Precision Acoustics, Dorchester, UK) was placed at the same position after the tests. The output of the function generator was a 10-cycle 5-Vpp burst waveform, the frequency of which was swept from 10 to $30 \mathrm{MHz}$ in a step of $1 \mathrm{MHz}$ because the hydrophone used is only calibrated at these frequencies.

To further evaluate the receiving SNR of the transducer, the transmitter was driven by a negative voltage pulse of 5-ns pulse width and 150-V peak amplitude generated by a pulser/receiver (DPR500, Imaginant). The UST and the aUST were setup to detect the pulse waves at different positions along the beam axis. The SNR, which is defined as the ratio of the peak amplitude of the signal to the root-mean-square value of the noise, was computed using MATLAB (The MathWorks, Natick, MA, USA). 


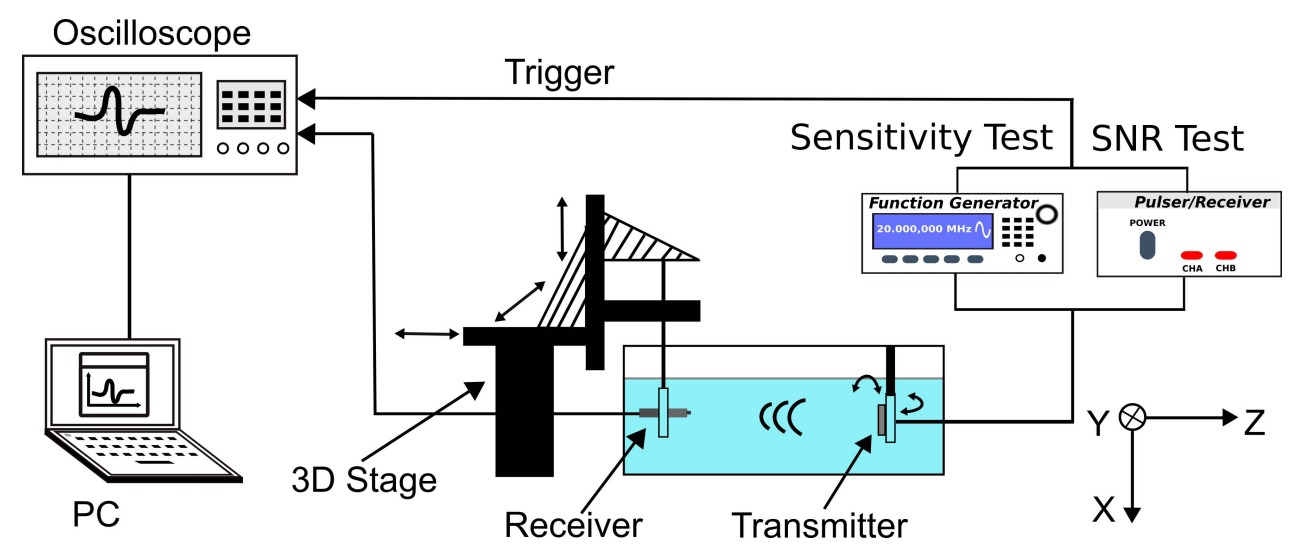

Figure 3. Schematic of the experimental setup of the receiving test. The transmitter is driven by the function generator during the sensitivity test and by the pulser-receiver during the SNR test.

\subsection{System Configuration}

A PA imaging system was developed to evaluate the imaging performance of the prototype, as shown in Figure 4. An optical parametric oscillator (OPO) laser (VIBRANT 532, OPOTEK, Carlsbad, CA, USA) with a tunable output was used to irradiate the sample with a laser pulse of 10-ns at a wavelength of $800 \mathrm{~nm}$ and a repetition rate of $10 \mathrm{~Hz}$. The laser beam was first reshaped by an iris (ID25, Thorlabs, Newton, NJ, USA) before being collimated by a double-lens system. The collimated laser beam was split into two beams via a pellicle beam splitter (BP108, Thorlabs, Newton, NJ, USA). One of them was directed to a fast photodiode (DET10A2, Thorlabs, Newton, NJ, USA) for monitoring the laser stability and triggering the oscilloscope. Another one was coupled to a multi-mode fiber (CSH1000, Shenzhen Xinrui Optical, Shenzhen, China) with a 1-mm core diameter using a microscope objective (RMS10X, Olympus, Tokyo, Japan). The output light from the fiber was focused on the sample by a fiber collimator (F220SMA-780, Thorlabs, Newton, NJ, USA) and an aspheric lens (A260TM-A, Thorlabs, Newton, NJ, USA), resulting in an illumination spot with a diameter of $\sim 1.4 \mathrm{~mm}$. The generated PA signals were detected by the UST and the aUST respectively, amplified by an amplifier with $55 \mathrm{~dB}$ gain (CLC-10K0.5G-5510-S, Connphy, Baltimore, MD, USA), and captured using an oscilloscope (MDO3022, Tektronix, Beaverton, OR, USA). An imaging head composed of the fiber collimator and the transducer was mounted on a linear stage (SC3002B, Zolix, Beijing, China) for imaging. To acquire a B-scan image, the imaging head was scanned along the $X$-axis with a step size of $100 \mu \mathrm{m}$. The whole imaging process including the move of the stage, the emitting of the laser and the data acquisition, was controlled by MATLAB software.

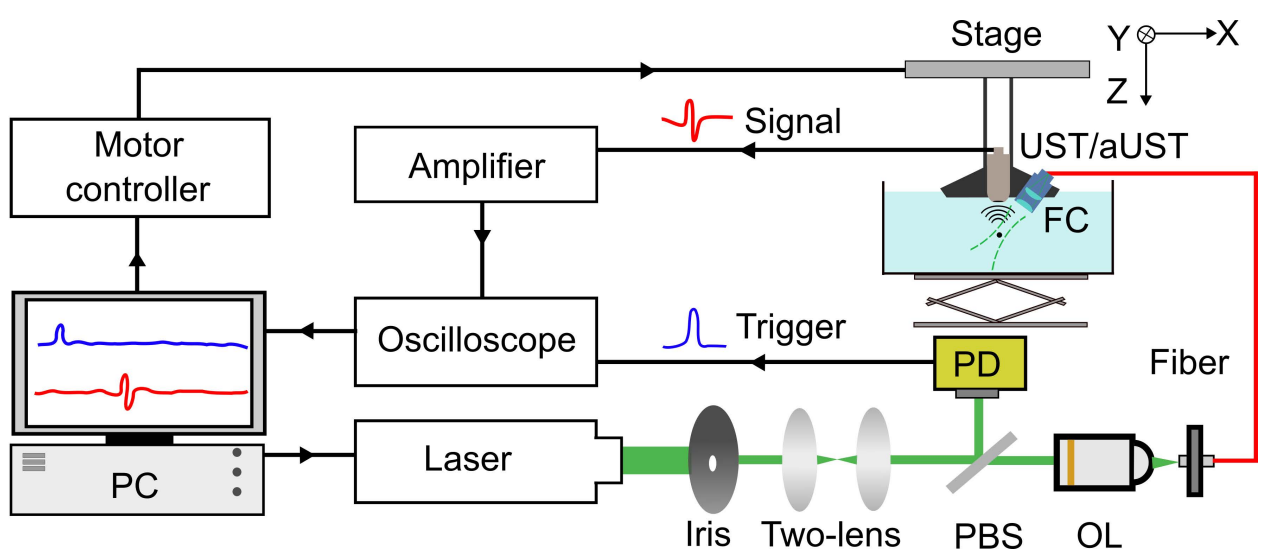

Figure 4. Schematic of the photoacoustic (PA) imaging system. PBS, pellicle beam splitter; OL, objective lens; PD, photodiode; FC, fiber collimator; UST: transducer without an integrated amplifier and aUST: transducer with an integrated amplifier. 


\subsection{Phantom Preparation}

A 0.5-mm pencil lead fixed on a mounting plate using UV (Ultraviolet) gel was used to demonstrate the enhanced detection of PA signals using the transducer with an integrated front-end amplifier. Two wire phantoms were prepared for evaluation of the spatial resolutions and the imaging depth range. The first wire phantom consisted of five $50-\mu \mathrm{m}$ tungsten wires attached on a U-shape holder. The wires were aligned in parallel with the $\mathrm{Y}$-axis and spaced $\sim 0.5 \mathrm{~mm}$ along the Z-axis. The second wire phantom had a 30- $\mu \mathrm{m}$ and a 50- $\mu \mathrm{m}$ tungsten wire crossing each other in the XZ plane. All wires were immersed in water for ultrasonic coupling. In vitro experiments were performed on a chicken breast tissue phantom with five $200-\mu \mathrm{m}$ wires embedded under the skin surface at depths ranging from 0.2 to $0.9 \mathrm{~mm}$.

\subsection{Data Processing}

PA signals emitted from samples were digitized at a sampling rate of $100 \mathrm{MS} / \mathrm{s}$ and averaged 8 times before being stored in a PC. The number of averaging was decreased compared to the before-mentioned 16 times averaging to reduce the acquisition time. The data were analyzed offline using MATLAB. Prior to image reconstruction, a band-pass filter with cut-off frequencies between 2 and $35 \mathrm{MHz}$ was applied to the original signals to eliminate out-of-band noise and aliasing. Then, the time-resolved PA signals were converted to depth-resolved A-lines based on the time of flight (ToF) principle. To make the image finer and improve the accuracy of Gauss fitting, each A-line was interpolated by a factor of 5 corresponding to $500 \mathrm{MS} / \mathrm{s}$ sampling rate. A B-scan image was reconstructed by stacking Hilbert transformed A-lines along the scan direction.

\section{Results and Discussion}

\subsection{Transducer Characterization}

Figure 5a shows the electrical impedance of the PZT element. The center frequency was measured to be $18 \mathrm{MHz}$. Figure $5 \mathrm{~b}$ shows the measured output frequency response of the front-end amplifier circuits. The measured $-3 \mathrm{~dB}$ bandwidth ranges from 5 to $200 \mathrm{MHz}$, making the amplifier suitable for detection of the wideband PA signals. Notably, the amplifier shows a flat response of over $25 \mathrm{~dB}$ gain from 10 to $50 \mathrm{MHz}$, which can completely cover the bandwidth of the fabricated PZT transducer. Figure $5 c$ shows the output noise voltage spectrum of the transducer with an integrated amplifier. The noise density at $20 \mathrm{MHz}$ was $14.9 \mathrm{nV} / \sqrt{ } \mathrm{Hz}$ and the integrated noise level from 2 to $35 \mathrm{MHz}$ was $138 \mu \mathrm{V}$.

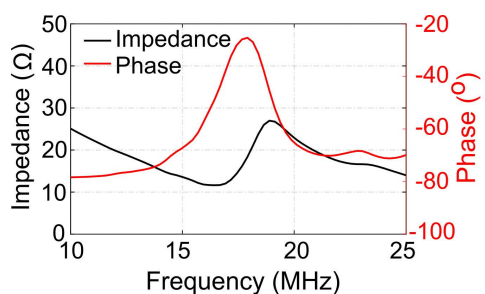

(a)

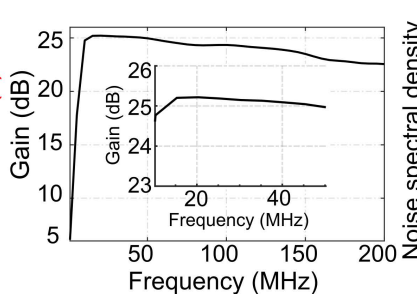

(b)

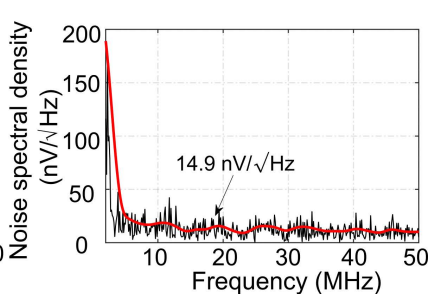

(c)

Figure 5. (a) Electrical impedance of the PZT transducer. (b) Output frequency response of the amplifier circuits. Inset: Output frequency response from 10 to $50 \mathrm{MHz}$. (c) Output noise spectral density of the transducer with an integrated amplifier.

Figure $6 \mathrm{a}, \mathrm{b}$ presents the typical received signals under a 10-cycle burst at $20 \mathrm{MHz}$ using the UST and the aUST, respectively. The peak-to-peak amplitude of the signal detected by the aUST was about 15 -fold higher than that detected by the UST. The amplification of the signal shows an agreement with the frequency response of the amplification circuits mentioned before. By dividing the calibrated acoustic pressure by the amplitude of the detected signal, the receiving sensitivity across 
the bandwidth of $10 \mathrm{MHz}$ to $30 \mathrm{MHz}$ was obtained. As shown in Figure 6c, the aUST achieved a significantly enhanced sensitivity of $62.1 \mu \mathrm{V} / \mathrm{Pa}$ at $20 \mathrm{MHz}$ compared with that of the UST $(4.3 \mu \mathrm{V} / \mathrm{Pa}$ at $20 \mathrm{MHz}$ ). In comparison, Chen et al. demonstrated a AIN-based piezoelectric transducer with the sensitivity of $4.22 \mu \mathrm{V} / \mathrm{Pa}$ [48]. As previously stated, the noise equivalent pressure (NEP) is computed by dividing the output noise by the receiving sensitivity of the transducer [49]. According to the results shown in Figure 6c, the NEP of the aUST was determined to be $0.24 \mathrm{mPa} / \sqrt{ } \mathrm{Hz}$ at $20 \mathrm{MHz}$.

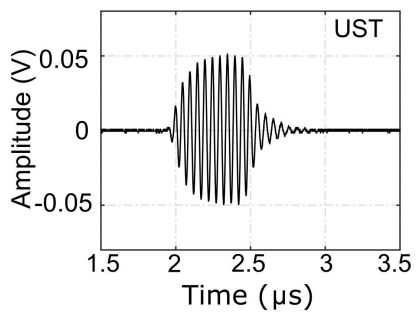

(a)

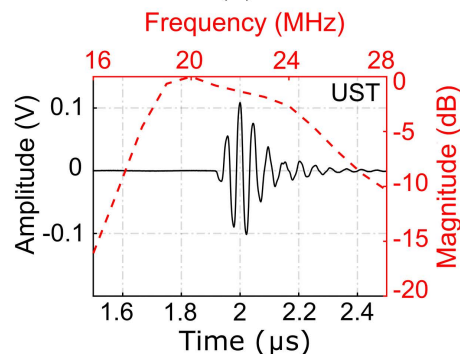

(d)

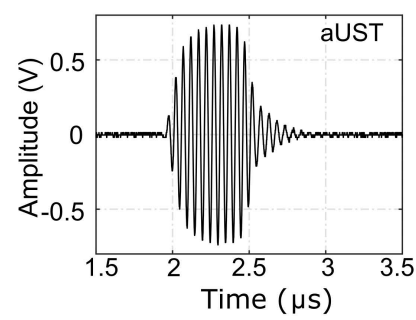

(b)

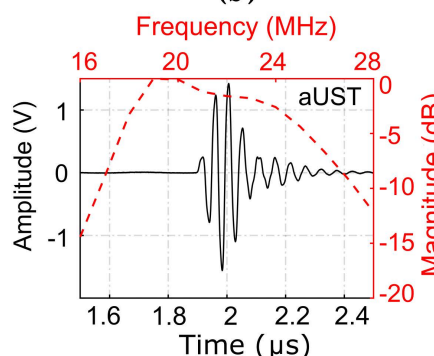

(e)

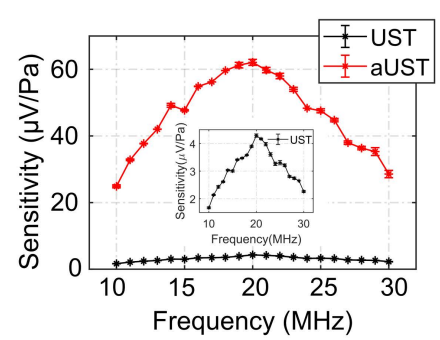

(c)

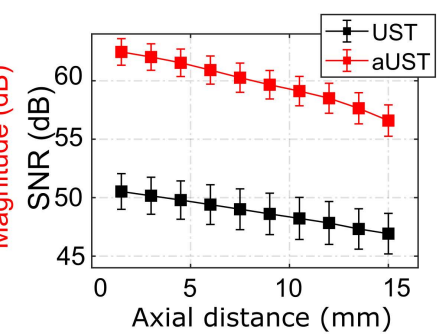

(f)

Figure 6. Performance characterization. (a-b) Typical received signals under a 10-cycle burst at $20 \mathrm{MHz}$ using the UST and the aUST, respectively. (c) Calibrated sensitivity. Insert: sensitivity of the UST. (d-e) Typical pulse signals received at $\sim 3 \mathrm{~mm}$ using the UST and the aUST, respectively. Red line: frequency spectrum; black line: pulse signal. (f) Signal-to-noise ratios (SNRs) along the beam axis. The graphs show results from three separate tests and the data are shown as means \pm SD. UST: transducer without integrated amplifier and aUST: transducer with an integrated amplifier.

Due to the flat response of the amplifier over the frequency range of $10-50 \mathrm{MHz}$, the $-6 \mathrm{~dB}$ bandwidth and the center frequency of the signal detected by the aUST (Figure 6d) present no significant changes compared with that detected by the UST (Figure 6e). The receiving performance of both transducers is summarized in Table 1. It should be noted that the bandwidth mentioned here is not the detection bandwidth of the transducer under test, because the received signal is the convolution of the impulse response of the receiver and the transmitted signal. The SNRs of the received signals detected by the aUST were improved by at least $10 \mathrm{~dB}$ compared with that detected by the UST (Figure 6f). Both transducers achieved decreasing SNRs along the beam axis due to the divergence of the acoustic beam. As shown in Table 1, the NEP achieved by the aUST was $\sim 9 \mathrm{~dB}$ lower than that achieved by the UST, which well explains the measured SNR improvement considering the imperfect alignment during the measurements. These results showcase that integrating front-end amplifier to the transducer is an effective method to improve the detection sensitivity of the acoustic detector while maintaining the bandwidth. The achieved high sensitivity will benefit PA signal detection under low energy depositions.

Table 1. Summary of the receiving performance.

\begin{tabular}{|c|c|c|c|c|c|c|}
\hline \multirow[b]{2}{*}{ Transducers } & \multicolumn{6}{|c|}{ Specifications } \\
\hline & Pulse Width (ns) & Center Frequency (MHz) & -6 dB Bandwidth (\%) & SNR at $3 \mathrm{~mm}(\mathrm{~dB})$ & $\begin{array}{c}\text { Sensitivity at } 20 \\
\mathrm{MHz}(\mu \mathrm{V} / \mathrm{Pa})\end{array}$ & $\begin{array}{c}\mathrm{NEP} \text { at } 20 \mathrm{MHz} \\
(\mathrm{mPa} / \sqrt{ } \mathrm{Hz})\end{array}$ \\
\hline UST & 99.8 & 19.75 & 41.6 & 50.5 & 4.3 & 0.64 \\
\hline
\end{tabular}




\subsection{Enhanced Detection of the PA Signal}

To evaluate the enhancement of detected PA signals, the pencil lead was illuminated under different light fluences and the generated PA signals were received using the UST and the aUST, separately. When switching from the UST to the aUST, the experimental configurations, including the laser energy and the position of the phantom, remained the same. The depth position of the aUST was adjusted until the detected PA signals had the same flight time to the UST. Therefore, it could be assured that the two transducers were placed in the same position during the measurements. The output energy of the laser was adjusted by changing the Q-switch delay and attenuated by the iris. To compensate for the fluctuation of the laser output, the amplitude of the PA signal was corrected by the laser energy per pulse. Figure 7a,b present the typical PA signals detected by the UST and the aUST under $56 \mathrm{~nJ}$ per pulse. The amplitude of the PA signal is enhanced by approximately 14 -fold using the aUST, which accords well with the results shown in Figure 6. In comparison with the UST, the aUST improved the SNR by $\sim 10 \mathrm{~dB}$ under different laser energies (Figure 7c). When the light fluence decreased, the aUST still showed satisfying SNR because of the enhanced detection sensitivity. The receiving bandwidth of the transducer with an integrated amplifier was measured using a broadband PA signal generated from a $10-\mu \mathrm{m}$ wire. According to Figure $7 \mathrm{~d}$, the transducer had a $-6 \mathrm{~dB}$ fractional bandwidth of $63 \%$. The full width at half magnitude (FWHM) of the Hilbert transformed envelope of the PA signal was measured to be $105 \mu \mathrm{m}$ based on the sound velocity of $1500 \mathrm{~m} / \mathrm{s}$ in water, which corresponds to the axial resolution of the transducer.

(a)

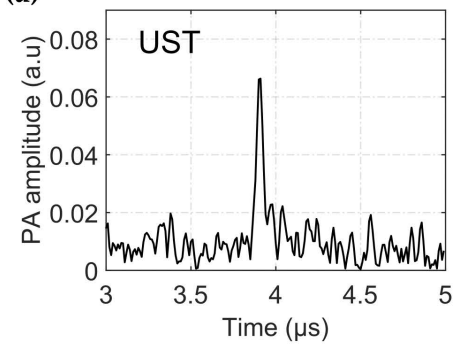

(c)

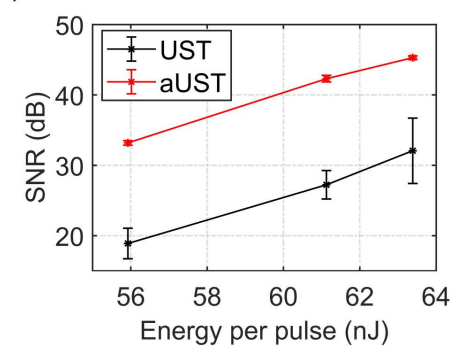

(b)

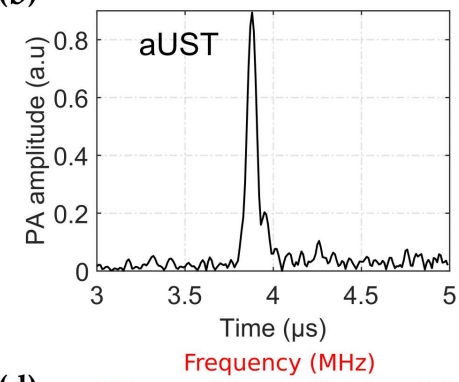

(d)

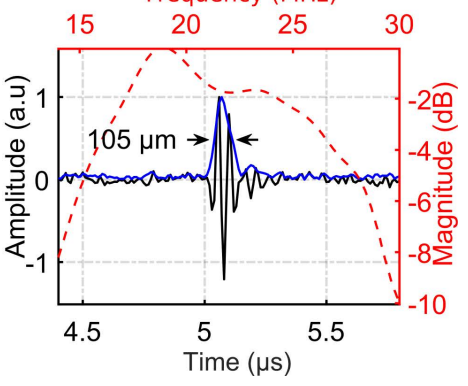

Figure 7. Enhanced detection of PA signals. (a-b) Envelope of the typical signal detected by the UST and the aUST respectively, under an optical fluence of $\sim 56 \mathrm{~nJ}$ per pulse. The range of the amplitude axis in (a) is set to one tenth of that in (b) for better illustration. (c) Signal-to-noise ratios (SNRs) under different light fluences. The graphs show results from three separate tests and the data are shown as means \pm SD. (d) Frequency response of the transducer with an integrated amplifier. UST: transducer without integrated amplifier and aUST: transducer with an integrated amplifier.

\subsection{Imaging of Wire Phantoms}

We evaluated the spatial resolution and the imaging depth range of the fabricated prototype using a phantom containing five $50-\mu \mathrm{m}$ tungsten wires aligned in a depth range of $\sim 2.5 \mathrm{~mm}$ with spacings of $\sim 0.5 \mathrm{~mm}$, as shown in Figure 8a. For comparison, the same area was imaged using the UST. Figure $8 \mathrm{c}$ presents the B-scan image acquired by the UST. Three wires located within the center of the illumination spot are clearly shown on the image, while the other two out-of-focus wires cannot be 
observed. Since a focused light was used in our system to illuminate the sample, wires placed in the optical focus absorb more laser energy and generate stronger signals than the wires away from the focus. This is further explained by the Hilbert-transformed envelope of the PA signal along the red dashed line in the B-scan image. Signals emitted from the central three wires could be well discriminated, while signals generated from other two wires were emerged in the noise floor. Compared with the UST, the aUST was able to detect all five wires with improved contrast (Figure 8d), corresponding to an enlarged imaging depth range of $\sim 2.5 \mathrm{~mm}$. The contrast-to-noise ratio (CNR) of the B-scan image was calculated as the ratio of the difference between the mean amplitude of the signal and the noise, to the root-mean-square value of the background noise area taken from the image [50]. Figure $8 \mathrm{~b}$ presents the CNRs at five depths from 4.8 to $7.3 \mathrm{~mm}$. There was a significant improvement of the CNR by more than $10 \mathrm{~dB}$ obtained by the aUST. Since the sample was illuminated with an angle of $45^{\circ}$ and the focus was placed $\sim 6 \mathrm{~mm}$ under the transducer, the CNR peaks at $\sim 6 \mathrm{~mm}$ depth and decreased as the wires were away from the optical focus. The slight differences between the graphs of the UST and the aUST were attributed to the non-identical directivity of the transducer and the slight position deviations during scanning.

(a)
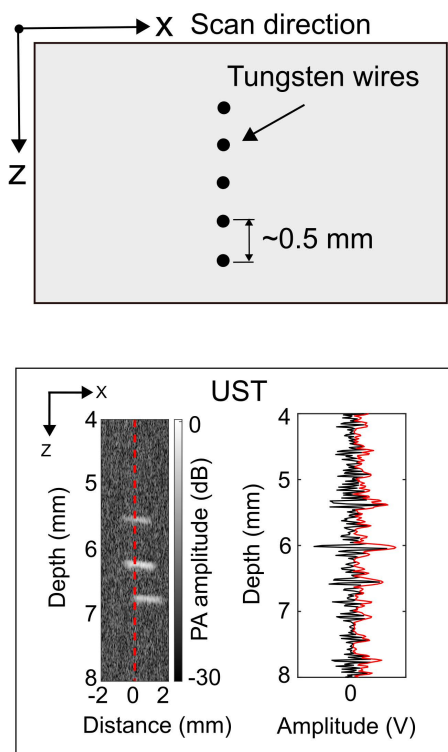

(b)

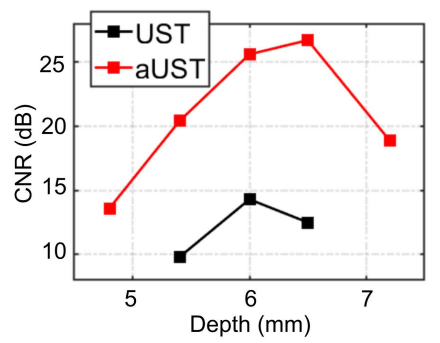

(e)

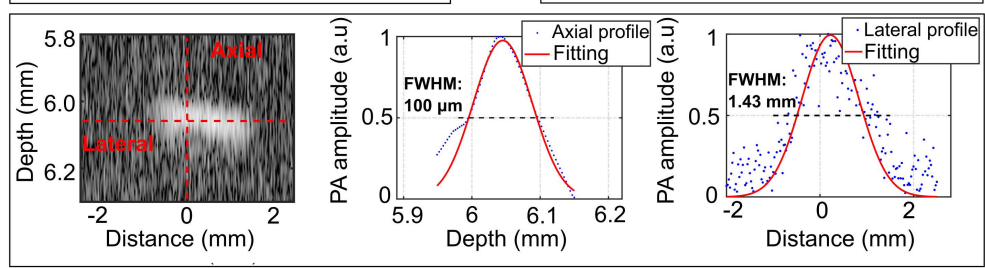

(f)
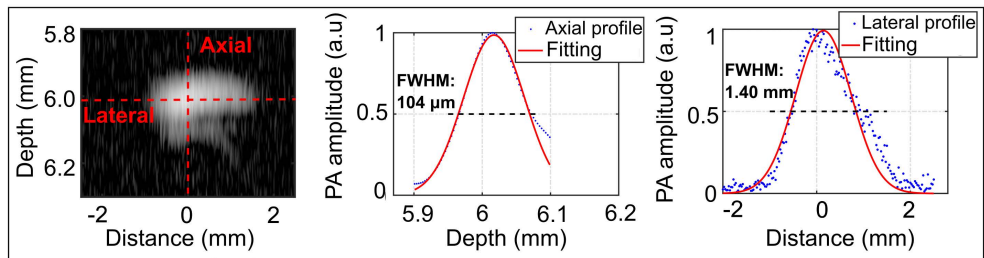

Figure 8. PA imaging of five tungsten wires.

(a) Schematic of the alignment of the wires. (b) Contrast-to-noise ratio (CNR) along depth. (c) B-scan image of five tungsten wires acquired by the UST at $30 \mathrm{~dB}$ dynamic range and the PA signal along the red dashed line. (d) B-scan image of five tungsten wires acquired by the aUST at $30 \mathrm{~dB}$ dynamic range and the PA signal along the red dashed line. (d,e) Axial and lateral resolution at a representative depth of $6 \mathrm{~mm}$ by the UST. (f) Axial and lateral resolution at a representative depth of $6 \mathrm{~mm}$ by the aUST. UST: transducer without integrated amplifier and aUST: transducer with an integrated amplifier. 
The wire reconstructed at $\sim 6 \mathrm{~mm}$ was used to estimate the spatial resolutions. Gaussian fitted profiles of the Hilbert transformed PA envelopes were computed using the Curve Fitting Toolbox in MATLAB. The resolution is defined as the FWHM of the fitted curve. As illustrated in Figure 8f, the axial resolution of the aUST was measured to be $104 \mu \mathrm{m}$, which is in good agreement with the result shown in Figure $7 \mathrm{~d}$. Both transducers achieve comparable axial resolutions because the integrated amplifier did not change the bandwidth significantly, as summarized in Table 1 . The lateral resolution of the current system was mainly dominated by the size of the optical beam at the focal plane, since the sensitivity field of the unfocused transducer was larger than the optical focus. To better show the improvement of the imaging depth range, we used a multi-mode fiber to achieve a large illumination spot of $1.4 \mathrm{~mm}$ in diameter.

To further demonstrate the imaging performance of the transducer, a wire phantom consisting of two crossing wires with diameters of $30 \mu \mathrm{m}$ and $50 \mu \mathrm{m}$ was imaged using the UST and the aUST, respectively. Figure 9a illustrates the experimental setup. The angle between two wires was small enough to assure that both wires were within the light spot. Due to low optical absorption, PA signals emitted from the $30-\mu \mathrm{m}$ wire were too weak to be detected by the UST, thus the $30-\mu \mathrm{m}$ wire could be barely identified in the B-scan image, as shown in Figure 9b. In comparison, both wires were clearly observed in Figure $9 \mathrm{c}$ with superior contrast, indicating the improved detection sensitivity of the aUST. The crossing point was not clearly distinguished because of two reasons: firstly, signals from two wires are strongly interfered when they get closer, resulting in an overlapped Hilbert transformed PA envelope and secondly, the axial resolution of the system is measured to be $\sim 104 \mu \mathrm{m}$, thus when the distance of two wires comes to this range, they cannot be resolved. A possible solution to this problem is to use a transducer of higher frequency and broader bandwidth to achieve finer axial resolution. Figure $9 \mathrm{~d}$ presents the CNRs at four locations labeled by P1-P4 in Figure 10c. The CNR of the UST at P1 is not available because the wire at this position cannot be recognized. In comparison, the CNRs were improved by $\sim 10 \mathrm{~dB}$ using the aUST, which agreed well with the before-mentioned results. The results of wire phantoms imaging experiments demonstrated that the transducer with an integrated front-end amplifier was capable of improving the image quality and depth of PA imaging by offering enhanced detection sensitivity.

(a)

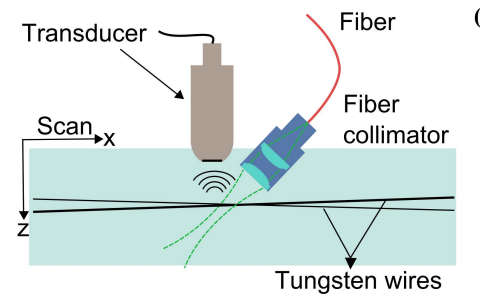

(d)

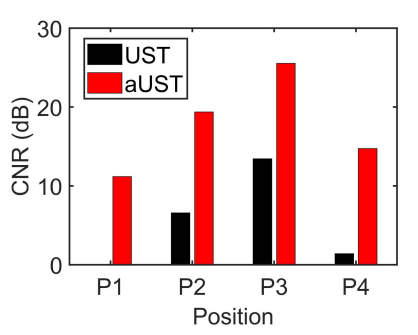

(b)

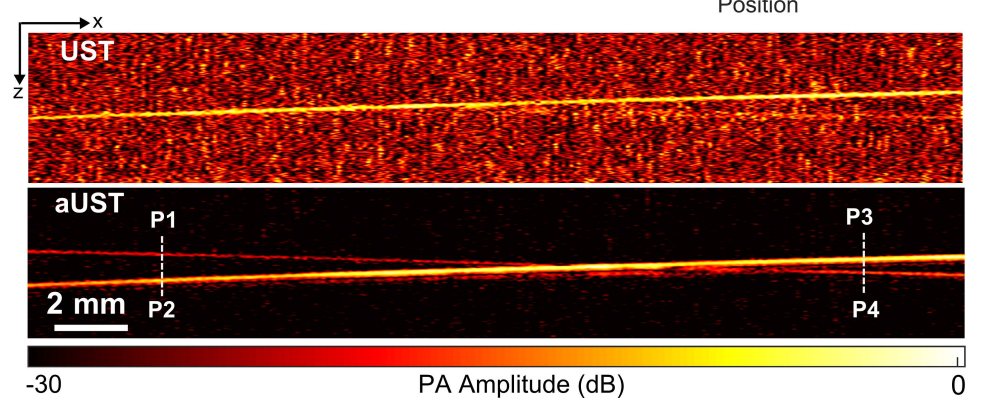

Figure 9. PA imaging of two crossing wires. (a) Schematic of the alignment of the transducer, the light source and the phantom. (b) B-scan image of two crossing wires acquired by the UST at $25 \mathrm{~dB}$ dynamic range. (c) B-scan image of two crossing wires acquired by the aUST at $30 \mathrm{~dB}$ dynamic range. (d) Contrast-to-noise ratios (CNRs) at four regions labeled by P1, P2, P3, and P4 in (c). The CNR of the UST at P1 is not available. UST: transducer without integrated amplifier and aUST: transducer with an integrated amplifier. 
(a)

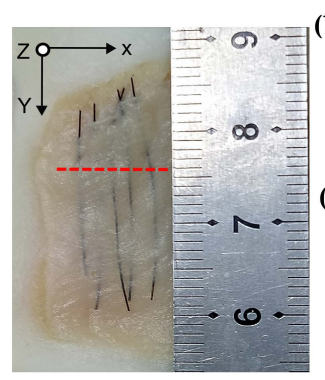

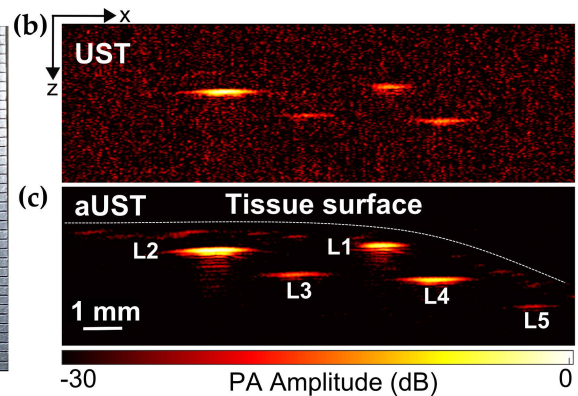

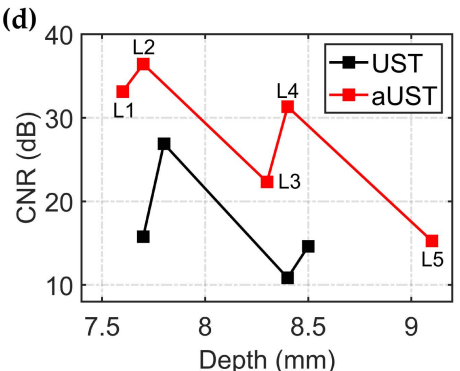

Figure 10. PA imaging of the chicken breast tissue phantom. (a) Photo of the chicken breast tissue phantom. Red dashed line represents the scanning plane. (b) B-scan image acquired by the UST at a $30 \mathrm{~dB}$ dynamic range. (c) B-scan image acquired by the aUST at a $30 \mathrm{~dB}$ dynamic range. (d) Contrast-to-noise ratios (CNRs) at different depths. CNR of UST at $\sim 9.1 \mathrm{~mm}$ is not available. UST: transducer without an integrated amplifier and aUST: transducer with an integrated amplifier.

\subsection{Imaging of Chicken Breast Tissue}

We also conducted in vitro experiments on chicken breast tissue phantom with five $200-\mu \mathrm{m}$ tungsten wires embedded under the tissue surface to further validate the imaging performance. For this experiment, the output laser energy from the fiber was limited to $850 \mathrm{~nJ}$ per pulse, thus the corresponding energy density deposited at the focus is calculated to be $\sim 55 \mu \mathrm{J} / \mathrm{cm}^{2}$, which is far lower than the laser safety limit for skin exposure $\left(32 \mathrm{~mJ} / \mathrm{cm}^{2}\right.$ at $\left.800 \mathrm{~nm}\right)$ outlined by ANSI [39]. We limited the laser energy to demonstrate that the prototype is capable of providing high CNR and deep imaging depth even at such low light fluence at which the conventional UST cannot obtain satisfying images.

Figure 10a shows the photo of the chicken breast tissue phantom and the red dashed line represents the scanning slice. Figure $10 \mathrm{~b}, \mathrm{c}$ present the B-scan images acquired by the UST and the aUST, respectively. In Figure 10c, all five wires, including L5 that is not shown in Figure 10b, were clearly visualized with high contrast and the distribution of the tissue surface could be well recognized. The distances of the embedded wires L1-L5 from the tissue surface were estimated to be $0.2 \mathrm{~mm}$, $0.5 \mathrm{~mm}, 0.9 \mathrm{~mm}, 0.7 \mathrm{~mm}$, and $0.5 \mathrm{~mm}$, respectively. The results suggest that it is possible to capture more anatomical structures using the transducer with an integrated amplifier due to the high detection sensitivity. The CNRs do not show uniform decreasing along the depth because of the uneven tissue surface. As shown in Figure 10d, although the wire L2 and L5 were located at similar depths under the surface, the CNR of L5 was actually much lower than that of L1. The reason is that the incident light was deflected by the tilted surface before going deeper in the tissue, resulting in low optical energy deposited on wire L5. Overall, the CNR of the PA image was improved by $10 \mathrm{~dB}$ using the aUST compared with that using UST, demonstrating the capability of sensitivity-enhanced PA imaging using the transducer with an integrated front-end amplifier.

\section{Conclusions}

In this work, a PZT transducer with an integrated front-end amplifier was developed to improve the detection sensitivity of PA imaging. High sensitivity is achieved by interfacing an amplifier die closely to the transducer to reduce the additional noise and provide signal amplification locally.

Acoustic characterization shows that the transducer with an integrated front-end amplifier had a fractional bandwidth of $63 \%$ and achieved a high detection sensitivity of $62.1 \mu \mathrm{V} / \mathrm{Pa}$ and a low NEP of $0.24 \mathrm{mPa} / \sqrt{ } \mathrm{Hz}$ at the central frequency of $20 \mathrm{MHz}$. Imaging experiments on wire phantoms and the chicken breast tissue demonstrated that the fabricated prototype was capable of improving the CNR by $10 \mathrm{~dB}$ and extending the imaging depth by $\sim 1 \mathrm{~mm}$. These results imply that integrating front-end amplifier to the transducer is a promising method for improving the detection sensitivity and has the potential for enhancement of image quality and penetration depth of PA imaging. 
The laser energy per pulse was adjusted to be hundreds of $n J$ to demonstrate that the transducer with an integrated front-end amplifier was able to obtain high contrast images at such low light fluence. It is possible to further improve the imaging depth by increasing the laser energy within the ANSI limits. The size of the transducer used in this work was not comparable to that reported previously $[12,15,16]$, which also limits the imaging depth range. The axial resolution achieved in this work was $104 \mu \mathrm{m}$. Since an unfocused transducer with a relative large aperture was used for acoustic detection, the lateral resolution of the current system $(1.4 \mathrm{~mm})$ was predominantly limited by the size of the illumination spot. As future work, a focused transducer or a tightly focused light will be used to achieve high-resolution PA imaging. The miniature size of the amplifier die and the compact integration scheme make the proposed method also possible for endoscopic application or array-based photoacoustic tomography where the size of the element is typically confined to a sub-millimeter.

In summary, coupling the front-end amplifier directly to the transducer is capable of improving the detection sensitivity and the penetration depth of PA imaging and may open up the possibility of small structures detection, weak physiological changes monitoring, and functional parameters measurements in deep tissue.

Author Contributions: Conceptualization, V.N., G.S. and Y.C.; methodology, C.Y., X.Z. and J.L.; software, Y.J.; validation, C.Y., X.J. and A.S.; formal analysis, C.Y.; investigation, Z.H.; resources, X.J.; data curation, C.Y. and Y.J.; writing - original draft preparation, C.Y.; writing - review and editing, A.S., G.S, X.J. and Y.C.; visualization, C.Y. and Y.J; supervision, V.N., G.S. and Y.C.; project administration, Y.C.; funding acquisition, X.J and Y.C. All authors have read and agreed to the published version of the manuscript.

Funding: This research was funded by the International Cooperation Program of Jiangsu Province (BZ2016023), the Jiangsu Provincial Key Research and Development Plan (BE2017601), and the Social Development Program of Jiangsu Province (BE2017674).

Conflicts of Interest: The authors declare no conflict of interest.

\section{References}

1. Ntziachristos, V. Going deeper than microscopy: the optical imaging frontier in biology. Nat. Methods 2010, 7, 603-614. [CrossRef]

2. Zackrisson, S.; van de Ven, S.M.W.Y.; Gambhir, S.S. Light In and Sound Out: Emerging Translational Strategies for Photoacoustic Imaging. Cancer Res. 2014, 74, 979-1004. [CrossRef]

3. Meng, J.; Song, L. Biomedical photoacoustics in China. Photoacoustics 2013, 1, 43-48. [CrossRef]

4. Taruttis, A.; Ntziachristos, V. Advances in real-time multispectral optoacoustic imaging and its applications. Nat. Photonics 2015, 9, 219-227. [CrossRef]

5. Choi, W.; Park, E.Y.; Jeon, S.; Kim, C. Clinical photoacoustic imaging platforms. Biomed. Eng. Lett. 2018, 8, 139-155. [CrossRef] [PubMed]

6. Valluru, K.S.; Chinni, B.K.; Rao, N.; Bhatt, S.; Dogra, V.S. Basics and Clinical Applications of Photoacoustic Imaging. Ultrasound Clin. 2009, 4, 403-429. [CrossRef]

7. Xia, J.; Yao, J.; Wang, L. Photoacoustic Tomography: Principles and Advances. Prog. Electromagn. Res. 2014, 147, 1-22. [CrossRef] [PubMed]

8. Steinberg, I.; Huland, D.M.; Vermesh, O.; Frostig, H.E.; Tummers, W.S.; Gambhir, S.S. Photoacoustic clinical imaging. Photoacoustics 2019, 14, 77-98. [CrossRef]

9. Zeng, L.; Piao, Z.; Huang, S.; Jia, W.; Chen, Z. Label-free optical-resolution photoacoustic microscopy of superficial microvasculature using a compact visible laser diode excitation. Opt. Express 2015, 23, 31026-31033. [CrossRef]

10. Zhang, E.; Laufer, J.G.; Pedley, R.B.; Beard, P.C. In vivo high-resolution 3D photoacoustic imaging of superficial vascular anatomy. Phys. Med. Biol. 2009, 54, 1035-1046. [CrossRef]

11. Zhang, H.; Maslov, K.; Li, M.; Stoica, G.; Wang, L. In vivo volumetric imaging of subcutaneous microvasculature by photoacoustic microscopy. Opt. Express 2006, 14, 9317-9323. [CrossRef]

12. Stylogiannis, A.; Prade, L.; Buehler, A.; Aguirre, J.; Sergiadis, G.; Ntziachristos, V. Continuous wave laser diodes enable fast optoacoustic imaging. Photoacoustics 2018, 9, 31-38. [CrossRef] [PubMed] 
13. Schwarz, M.; Aguirre, J.; Buehler, A.; Omar, M.; Ntziachristos, V. Visualization of the microcirculatory network in skin by high frequency optoacoustic mesoscopy. In Proceedings of the Opto-Acoustic Methods and Applications in Biophotonics II, Munich, Germany, 21-25 June 2015; p. 9539.

14. Wang, X.; Pang, Y.; Ku, G.; Xie, X.; Stoica, G.; Wang, L. Noninvasive laser-induced photoacoustic tomography for structural and functional in vivo imaging of the brain. Nat. Biotechnol. 2003, 21, 803-806. [CrossRef] [PubMed]

15. Qin, W.; Jin, T.; Guo, H.; Xi, L. Large-field-of-view optical resolution photoacoustic microscopy. Opt. Express 2018, 26, 4271-4278. [CrossRef] [PubMed]

16. Aguirre, J.; Schwarz, M.; Soliman, D.; Buehler, A.; Omar, M.; Ntziachristos, V. Broadband mesoscopic optoacoustic tomography reveals skin layers. Opt. Lett. 2014, 39, 6297-6300. [CrossRef] [PubMed]

17. Jansen, K.; Van Soest, G.; Van Der Steen, A.F.W. Intravascular photoacoustic imaging: a new tool for vulnerable plaque identification. Ultrason. Med. Biol. 2014, 40, 1037-1048. [CrossRef] [PubMed]

18. Hui, J.; Cao, Y.; Zhang, Y.; Kole, A.; Wang, P.; Yu, G.; Eakins, G.; Sturek, M.; Chen, W.; Cheng, J.-X. Real-time intravascular photoacoustic-ultrasound imaging of lipid-laden plaque in human coronary artery at 16 frames per second. Sci. Rep. 2017, 7, 1-11. [CrossRef]

19. Zhang, J.; Yang, S.; Ji, X.; Zhou, Q.; Xing, D. Characterization of Lipid-Rich Aortic Plaques by Intravascular Photoacoustic Tomography Ex Vivo and In Vivo Validation in a Rabbit Atherosclerosis Model With Histologic Correlation. J. Am. Coll. Cardiol. 2014, 64, 385-390. [CrossRef]

20. Bendinger, A.L.; Glowa, C.; Peter, J.; Karger, C.P. Photoacoustic imaging to assess pixel-based sO(2) distributions in experimental prostate tumors. J. Biomed. Opt. 2018, 23, 11. [CrossRef]

21. Wood, C.; Harutyunyan, K.; De La Cerda, J.; Kaffes, C.; Millward, N.Z.; Shanmugavelandy, S.; Konopleva, M.; Bouchard, R. Assessment of blood oxygen saturation using spectroscopic photoacoustic imaging as a biomarker for disease progression in a small-animal leukemia model. In Medical Imaging 2018: Ultrasonic Imaging And Tomography; International Society for Optics and Photonics: Houston, TX, USA, 2018; Volume 10580.

22. Zhang, H.F.; Maslov, K.; Stoica, G.; Wang, L.V. Functional photoacoustic microscopy for high-resolution and noninvasive in vivo imaging. Nat. Biotechnol. 2006, 24, 848-851. [CrossRef]

23. Cao, R.; Li, J.; Ning, B.; Sun, N.; Wang, T.; Zuo, Z.; Hu, S. Functional and oxygen-metabolic photoacoustic microscopy of the awake mouse brain. Neuroimage 2017, 150, 77-87. [CrossRef]

24. Larina, I.V.; Larin, K.V.; Esenaliev, R.O. Real-time optoacoustic monitoring of temperature in tissues. J. Phys. D Appl. Phys. 2005, 38, 2633-2639. [CrossRef]

25. Yao, J.; Ke, H.; Tai, S.; Zhou, Y.; Wang, L. Absolute photoacoustic thermometry in deep tissue. Opt. Lett. 2013, 38, 5228-5231. [CrossRef] [PubMed]

26. Landa, F.J.O.; Dean-Ben, X.L.; Sroka, R.; Razansky, D. Four-dimensional optoacoustic temperature mapping in laser-induced thermotherapy. In Photons Plus Ultrasound: Imaging And Sensing; International Society for Optics and Photonics: Houston, TX, USA, 2018.

27. Nie, L.; Chen, M.; Sun, X.; Rong, P.; Zheng, N.; Chen, X. Palladium nanosheets as highly stable and effective contrast agents for in vivo photoacoustic molecular imaging. Nanoscale 2014, 6, 1271-1276. [CrossRef] [PubMed]

28. Paproski, R.J.; Forbrich, A.; Harrison, T.; Hitt, M.; Zemp, R.J. Photoacoustic imaging of gene expression using tyrosinase as a reporter gene. In Photons Plus Ultrasound: Imaging and Sensing; International Society for Optics and Photonics: Houston, TX, USA, 2011; Volume 7899.

29. Paproski, R.J.; Heinmiller, A.; Wachowicz, K.; Zemp, R.J. Multi-wavelength photoacoustic imaging of inducible tyrosinase reporter gene expression in xenograft tumors. Sci. Rep. 2014, 4, 5329. [CrossRef] [PubMed]

30. Weber, J.; Beard, P.C.; Bohndiek, S.E. Contrast agents for molecular photoacoustic imaging. Nat. Methods 2016, 13, 639-650. [CrossRef]

31. Liu, W.; Shcherbakova, D.M.; Kurupassery, N.; Li, Y.; Zhou, Q.F.; Verkhushaz, V.V.; Yao, J.J. Quad-mode functional and molecular photoacoustic microscopy. Sci. Rep. 2018, 8. [CrossRef]

32. Ji, X.R.; Xiong, K.D.; Yang, S.H.; Xing, D. Intravascular confocal photoacoustic endoscope with dual-element ultrasonic transducer. Opt. Express 2015, 23, 9130-9136. [CrossRef]

33. Galanzha, E.I.; Shashkov, E.V.; Kelly, T.; Kim, J.W.; Yang, L.; Zharov, V.P. In vivo magnetic enrichment and multiplex photoacoustic detection of circulating tumour cells. Nat. Nanotechnol. 2009, 4, 855-860. [CrossRef] 
34. Mehrmohammadi, M.; Yoon, S.J.; Yeager, D.; Emelianov, S.Y. Photoacoustic Imaging for Cancer Detection and Staging. Curr. Mol. Imaging 2013, 2, 89-105. [CrossRef]

35. Beard, P. Biomedical photoacoustic imaging. Interface Focus 2011, 1, 602-631. [CrossRef] [PubMed]

36. Rosenthal, A.; Razansky, D.; Ntziachristos, V. High-sensitivity compact ultrasonic detector based on a pi-phase-shifted fiber Bragg grating. Opt. Lett. 2011, 36, 1833-1835. [CrossRef] [PubMed]

37. Hoskins, P.; thrush, a.; Martin, K.; Whittingham, T. Diagnostic ultrasound: physics and equipment; CRC Press: Boca Raton, FL, USA, 2003.

38. Yao, J.; Wang, L.V. Sensitivity of photoacoustic microscopy. Photoacoustics 2014, 2, 87-101. [CrossRef] [PubMed]

39. White, I.J.; Dederich, H.D. American National Standard for the Safe Use of Lasers, ANSI Standard Z 136.1 2007; Laser Institute of America: Orlando, FL, USA, 2007.

40. Chan, J.; Zheng, Z.; Bell, K.; Le, M.; Reza, P.H.; Yeow, J.T.W. Photoacoustic Imaging with Capacitive Micromachined Ultrasound Transducers: Principles and Developments. Sensors 2019, 19, 3617. [CrossRef]

41. Lutzweiler, C.; Razansky, D. Optoacoustic Imaging and Tomography: Reconstruction Approaches and Outstanding Challenges in Image Performance and Quantification. Sensors 2013, 13, 7345-7384. [CrossRef]

42. Chen, C.; Raghunathan, S.B.; Yu, Z.; Shabanimotlagh, M.; Chen, Z.; Chang, Z.; Blaak, S.; Prins, C.; Ponte, J.; Noothout, E.; et al. A Prototype PZT Matrix Transducer With Low-Power Integrated Receive ASIC for 3-D Transesophageal Echocardiography. IEEE Trans. Ultrason. Ferroelectr. Freq. Control 2016, 63, 47-59. [CrossRef]

43. Wildes, D.; Lee, W.; Haider, B.; Cogan, S.; Sundaresan, K.; Mills, D.M.; Yetter, C.; Hart, P.H.; Haun, C.R.; Concepcion, M.; et al. 4-D ICE: A 2-D Array Transducer With Integrated ASIC in a 10-Fr Catheter for Real-Time 3-D Intracardiac Echocardiography. IEEE Trans. Ultrason. Ferroelectr. Freq. Control 2016, 63, 2159-2173. [CrossRef]

44. Moini, A.; Nikoozadeh, A.; Choe, J.W.; Chang, C.; Stephens, D.N.; Sahn, D.J.; Khuri-Yakub, P.T. Fully Integrated 2D CMUT Ring Arrays for Endoscopic Ultrasound. In Proceedings of the 2016 IEEE International Ultrasonics Symposium (IUS), Tours, France, 18-21 September 2016.

45. Jeon, S.; Kim, J.; Lee, D.; Baik, J.W.; Kim, C. Review on practical photoacoustic microscopy. Photoacoustics 2019, 15, 100141. [CrossRef]

46. Yang, C.; Jian, X.; Zhu, X.; Lv, J.; Han, Z.; Sergiadis, G.; Cui, Y. Highly sensitive PZT transducer with integrated miniature amplifier for photoacoustic imaging. In Proceedings of the 2019 IEEE International Ultrasonics Symposium (IUS), Galsgow, UK, 6-9 October 2019; pp. 981-984.

47. Hicks, B.; Erickson, B. Bias-T Design Considerations for LWA. LWA Memo 135. 2018. Available online: http://www.ece.vt.edu/swe/lwa (accessed on 29 April 2008).

48. Chen, B.; Chu, F.; Liu, X.; Li, Y.; Rong, J.; Jiang, H. AlN-based piezoelectric micromachined ultrasonic transducer for photoacoustic imaging. Appl. Phys. Lett. 2013, 103, 031118. [CrossRef]

49. Wygant, I.O.; Zhuang, X.; Yeh, D.T.; Oralkan, O.; Ergun, A.S.; Karaman, M.; Khuri-Yakub, B.T. Integration of 2D CMUT arrays with front-end electronics for volumetric ultrasound imaging. IEEE Trans. Ultrason. Ferroelectr. Freq. Control 2008, 55, 327-342. [CrossRef]

50. Vallet, M.; Varray, F.; Boutet, J.; Dinten, J.M.; Caliano, G.; Savoia, A.S.; Vray, D. Quantitative comparison of PZT and CMUT probes for photoacoustic imaging: Experimental validation. Photoacoustics 2017, 8, 48-58. [CrossRef] [PubMed]

(C) 2020 by the authors. Licensee MDPI, Basel, Switzerland. This article is an open access article distributed under the terms and conditions of the Creative Commons Attribution (CC BY) license (http://creativecommons.org/licenses/by/4.0/). 